\title{
On the intensity contrast of solar photospheric faculae and network elements
}

\author{
A. Ortiz ${ }^{1}$, S. K. Solanki ${ }^{2}$, V. Domingo ${ }^{1,3}$, M. Fligge ${ }^{4}$, and B. Sanahuja ${ }^{1,3}$ \\ 1 Departament d'Astronomia i Meteorologia, Universitat de Barcelona, Martí i Franquès 1, \\ 08028 Barcelona, Spain \\ 2 Max-Planck-Institut für Aeronomie, Max-Planck-Str. 2, 37191 Katlenburg-Lindau, Germany \\ 3 Institut d'Estudis Espacials de Catalunya, Gran Capità 2-4, 08034 Barcelona, Spain \\ ${ }^{4}$ Institute of Astronomy, ETH-Zentrum, Scheuchzerstr. 7, 8092 Zürich, Switzerland
}

Received 21 December 2001 / Accepted 28 March 2002

\begin{abstract}
Sunspots, faculae and the magnetic network contribute to solar irradiance variations. The contribution due to faculae and the network is of basic importance, but suffers from considerable uncertainty. We determine the contrasts of active region faculae and the network, both as a function of heliocentric angle and magnetogram signal. To achieve this, we analyze near-simultaneous full disk images of photospheric continuum intensity and lineof-sight magnetic field provided by the Michelson Doppler Interferometer (MDI) on board the SOHO spacecraft. Starting from the surface distribution of the solar magnetic field we first construct a mask, which is then used to determine the brightness of magnetic features, and the relatively field-free part of the photosphere separately. By sorting the magnetogram signal into different bins we are able to distinguish between the contrasts of different concentrations of magnetic field. We find that the center-to-limb variation (CLV) of the contrast changes strongly with magnetogram signal. Thus, the contrasts of active region faculae (large magnetogram signal) and the network (small signal) exhibit a very different CLV, showing that the populations of magnetic flux tubes that underly the two kinds of features are different. The results are compatible with, on average, larger flux tubes in faculae than in the network. This implies that these elements need to be treated separately when reconstructing variations of the total solar irradiance with high precision. We have obtained an analytical expression for the contrast of photospheric magnetic features as a function of both position on the disk and spatially averaged magnetic field strength, by performing a 2-dimensional fit to the observations. We also provide a linear relationship between magnetogram signal and the $\mu=\cos (\theta)$, where $\theta$ is the heliocentric angle, at which the contrast is maximal. Finally, we show that the maximum contrast per unit magnetic flux decreases rapidly with increasing magnetogram signal, supporting earlier evidence that the intrinsic contrast of magnetic flux tubes in the network is higher.
\end{abstract}

Key words. Sun: activity - Sun: faculae, plages - Sun: magnetic fields

\section{Introduction}

Radiometers on board satellites launched during the last three decades (NIMBUS-7, SMM, UARS, EURECA, $\mathrm{SOHO}$ ) have revealed that the total solar irradiance, also referred to as the solar constant, changes on a variety of time-scales. Solar irradiance variations on scales of days up to the solar activity cycle length are closely related to the evolution of the solar surface magnetic field, because the emergence and evolution of active regions (AR) on the solar surface is reflected in the irradiance records (Lean et al. 1998; Fligge \& Solanki 2000b). Sunspots and active region faculae are considered to be the dominant contributors to solar irradiance changes on time-scales of days to weeks. Space-based irradiance records have

Send offprint requests to: A. Ortiz,

e-mail: aortiz@am.ub.es also established a variation of about $0.1 \%$ of the irradiance in phase with the 11 year solar activity cycle, giving as a result a brighter Sun around activity maximum (Chapman 1987; Willson \& Hudson 1988). The origin of the long-term increase of the irradiance between activity minimum and maximum is still widely debated. It is expected that small-scale magnetic elements that compose the enhanced and quiet network contribute substantially to the observed irradiance increase during activity maximum (Foukal \& Lean 1988; Solanki \& Fligge 2001; Fligge \& Solanki 2000a). Nevertheless, other mechanisms of nonmagnetic origin have also been proposed, based, for example, on temporal changes in the latitude-dependent surface temperature of the Sun (Kuhn et al. 1988). Other authors have tried to explain these variations by modelling structural changes in the convection zone during the solar cycle (Balmforth et al. 1996). 
The photospheric magnetic field is concentrated in discrete elements whose diameters range from less than a hundred to several tens of thousands of kilometers. The brightness signature of these magnetic features is a strong function of their heliocentric angle and their size; sunspots are dark while small flux tubes are generally bright; faculae appear brighter near the limb (Solanki 1993, 2001). However, our knowledge of the brightness of small scale magnetic features, groups of which form faculae and the network, is incomplete (e.g. Solanki 1994).

Faculae, bright structures seen in the photosphere cospatially with chromospheric plages, are associated with magnetic fields. At high resolution, they consist of many unresolved small continuum bright points, with diameters of about $100 \mathrm{~km}$, called facular points (Muller 1983; Berger et al. 1995). The observed zoo of small magnetic features is unifyingly described by the concept of the small flux tube. To predict their physical properties, different models for small flux tubes have been constructed (e.g., Spruit 1976; Deinzer et al. 1984a, 1984b; Knölker et al. 1988; Knölker \& Schüssler 1988; Grossmann-Doerth et al. 1989; Steiner et al. 1996). According to this model faculae are conglomerates of evacuated flux tubes with hot walls and a hot or cool floor (corresponding to an optical depth of $\tau=1$ ) depending on the evacuation and diameter of the flux tube. The model predicts a certain CLV of the contrast for a particular diameter of the underlying flux tubes.

This model assumes that inside each small flux tube the magnetic field is of the order of a kilogauss, but practically zero outside. Due to the magnetic pressure the fluxtube interior is evacuated, so that $\tau=1$ is reached along its walls (which are bright due to radiation leaking in from the surroundings, i.e., there is a horizontal flux of energy into the tube). Flux tubes can be dark at disk center if suppression of convective energy transport within the tube is included (e.g., Spruit 1976; Deinzer et al. 1984a, 1984b; Knölker et al. 1988), resulting in a cooling of the deeper layers. When the tube is sufficiently broad, the horizontal optical depth between the wall and the tube center is large and most of the radiation cannot reach the center. In this case, the tube floor remains dark at its center. But if the tube is sufficiently slender, the horizontal flux of energy can reach the center of the tube; then, the interior of the tube is heated, the vertical energy flux increases, and the tube turns bright, even at disk center. In this scenario, the transition between smaller bright points and larger dark micropores (e.g., Topka et al. 1997) occurs at a diameter of about $300 \mathrm{~km}$ (e.g., Grossmann-Doerth et al. 1994), and therefore micropores would fill the gap between small bright points and larger dark pores. Micropores are predominantly found in active regions, while bright points are the main constituents of the network. When observed near the limb, the heated walls of the tube become visible, and therefore the contrast increases.

Observations also provide evidence that the contrast, as well as the underlying thermal structure, depends on the size of the flux tubes (e.g., Keller 1992), but more commonly on the strength of the magnetogram signal
(Frazier 1971; Spruit \& Zwaan 1981; Solanki \& Stenflo 1984; Solanki 1986; Zayer et al. 1990; Solanki \& Brigljević 1992; Topka et al. 1992, 1997; Lawrence et al. 1993; Grossmann-Doerth et al. 1994), and hence a test of flux tube models is possible with such data. However, since most flux tubes are not resolved, it is necessary to have a well-defined and constant spatial resolution of the observations with which to compare the models. With groundbased data, the basis of practically all facular contrast CLV measurements to date, this criterion is hard to meet.

Differences in spatial resolution, caused by variable seeing, may indeed partly explain the variety of measured contrast CLVs (e.g., Libbrecht \& Kuhn 1984; Unruh et al. 1999). Other possible factors are differences in wavelength, spectral resolution and the magnetic filling factor of the observed features (Solanki 1994). The problem posed by variable seeing can be circumvented by employing data recorded in space, while the magnetic filling factor can be estimated with the help of cospatial and cotemporal magnetograms. Only relatively few contrast investigations including the magnetogram signal can be found in the literature (e.g. Frazier 1971; Foukal \& Fowler 1984; Topka et al. 1992, 1997; Lawrence et al. 1993).

Here we add to this list using data from the MDI instrument on board SOHO (Domingo et al. 1995); their main advantages are:

- seeing effects due to the Earth's atmosphere are avoided,

- measurements are made in the pure continuum (i.e. spectral resolution is not a problem),

- the 20-min averaged MDI magnetograms have a reasonably low noise level,

- a large and homogeneous data set is available,

- the characteristics of the instrument and the data sets are well known and stable,

- magnetograms and intensity images are obtained regularly by the same instrument with exactly the same spatial resolution, so that a one-to-one identification of brightness with magnetogram signals can be made.

The main disadvantages of the MDI data are:

$$
\begin{aligned}
& \text { - a relatively low spatial resolution with a pixel size of } \\
& 2 \times 2^{\prime \prime}, \\
& \text { - } \\
& \text { measurements are available at only a single wave- } \\
& \text { length. }
\end{aligned}
$$

The purpose of this paper is to present new high-quality measurements of the contrast of the photospheric bright features as a function of both heliocentric angle and magnetogram signal and to obtain an analytical function that predicts their contrast given a position on the disk and a magnetic signal value. Such measurements are expected to be of use not only to constrain models of flux tubes, but also to improve the modelling of the solar irradiance (Lean et al. 1998). Uncertainties in the contrast of faculae and the network are one of the major sources of error in the modelling of solar irradiance variations. Employing MDI 
data to obtain the contrast as input for irradiance modelling is of particular interest since MDI magnetograms have already been succesfully used for such modelling (Fligge \& Solanki 2000a).

In Sect. 2 we present the data sets used and the analysis procedures. In Sect. 3 we describe the results, which are discussed in Sect. 4. Finally, our conclusions and a summary are given in Sect. 5 .

\section{Data and analysis procedure}

\subsection{Data sets}

The Solar Oscillations Investigation/Michelson Doppler Imager (SOI/MDI) instrument is a state-of-the-art helioseismology experiment and magnetograph on board the SOHO spacecraft, devoted to study the interior structure and dynamics as well as the surface magnetic field of the Sun. This instrument gives an image of the Sun on a $1024 \times 1024$ CCD camera, and can observe in two spatial resolution modes, full disk and high-resolution of the central part of the disk (HR). We are interested in the full disk measurements, which have a field of view of $34 \times 34^{\prime}$ and a pixel size of $2 \times 2^{\prime \prime}$. Two tunable Michelson interferometers allow MDI to record filtergrams centered at five wavelengths across the Ni I $6768 \AA$ absorption line. From the filtergrams, MDI computes the following six observables: Doppler velocity, continuum intensity, line depth, longitudinal magnetic field, horizontal velocity and limb position. The SOI/MDI instrument is described in detail by Scherrer et al. (1995).

The products of interest for our work are the full disk magnetograms and continuum intensity images. Magnetograms only measure net magnetic flux per resolution element, therefore the signal is not the true magnetic field strength $\boldsymbol{B}$, inside a flux tube, but its longitudinal component, $\langle|\boldsymbol{B}| \cos \gamma\rangle$, averaged over the pixel, where $\gamma$ is the angle between the magnetic vector and the line of sight. For simplicity, we hereafter refer to $\langle|\boldsymbol{B}| \cos \gamma\rangle$ as $B$. In a 2-component model of the magnetic field, with magnetic flux tubes of field strength $\boldsymbol{B}$ covering a fraction $\alpha$ of the solar surface separated by a field-free component covering $(1-\alpha)$ of the surface, we can write $\langle|\boldsymbol{B}| \cos \gamma\rangle$ as $\alpha|\boldsymbol{B}| \cos \gamma$. Since the true field strength $|\boldsymbol{B}|$ lies in a relatively narrow range of 1000-1500 G for all magnetic features except intranetwork elements (Solanki et al. 1999), and $\cos \gamma \approx \cos \theta \approx \mu$ is a reasonable approximation $(\theta$ is the heliocentric angle), the strength of the magnetogram signal mainly provides information on the magnetic filling factor $\alpha$.

MDI magnetograms are usually obtained every $96 \mathrm{~min}$, with the exception of periodic campaigns in which 1-min cadence measurements are available. The 1- $\sigma$ noise level for a one-min longitudinal magnetogram is $20 \mathrm{G}$. Full disk continuum intensity images are taken each minute with a noise level of $0.3 \%$.

The analyzed data set consists of nearly simultaneous magnetograms and continuum intensity images recorded
Table 1. Selected days and times (hours $/ \mathrm{min} / \mathrm{sec}$ ) during 1999 at which the averaged magnetograms analyzed here were recorded (see text for details).

\begin{tabular}{ll}
\hline \hline 1999 observation dates & Time (UT) \\
\hline February 13 & $00: 10: 02$ \\
February 20 & $04: 10: 02$ \\
May 14 & $00: 10: 03$ \\
May 28 & $06: 10: 03$ \\
June 25 & $01: 10: 02$ \\
July 2 & $03: 10: 02$ \\
July 10 & $01: 10: 02$ \\
August 7 & $00: 10: 02$ \\
October 12 & $09: 10: 03$ \\
October 15 & $06: 10: 03$ \\
\hline
\end{tabular}

on 10 days in the period February to October, 1999, as shown in Table 1. The time of the observations is given for the averaged magnetograms (see Sect. 2.2). It corresponds to the middle of the 20 min integration time. These days were chosen because they belong to a high activity period so that everything from quiet network to intense activeregion plage was present on the solar surface. The sample contains active regions spread over almost all $\mu=\cos \theta$ values. They are also generally well separated in time in order to avoid duplication.

\subsection{Reduction method and analysis}

We employ averages over 20 single magnetograms, taken at a cadence of 1 per minute, in order to reduce the noise level sufficiently to reliably identify the quiet network. The individual magnetograms were rotated to compensate for the time difference before averaging. Intensities are standard 1-min images. Care has been taken to use intensity images obtained as close in time to the magnetograms as possible. In all cases but one, the two types of images were recorded within $30 \mathrm{~min}$ of each other, with $37 \mathrm{~min}$ being the highest difference. The intensity images have been rotated to co-align them with the corresponding average magnetogram. Intensity images have also been corrected for limb-darkening effects using a fifth order polynomial in $\mu$ following Neckel \& Labs (1994). Our final data sets are pairs of co-aligned averaged magnetograms and photospheric continuum intensity images for each of the 10 selected days. Both types of images can be compared pixel by pixel. An example magnetogram and the corresponding intensity image recorded on October 12, 1999 are shown in Fig. 1 (top and middle panels).

We have determined the noise level of the MDI magnetograms and continuum images as a function of position over the CCD array. The standard deviation for the magnetic signal has been calculated using a running $100 \times 100$ pixel box over the solar disk, with the exception of the limbs, which were avoided by masking out an outer ring of 75 pixels width. This process was applied to several 1996 low activity magnetograms, in order to avoid artifacts introduced by the presence of active regions. 

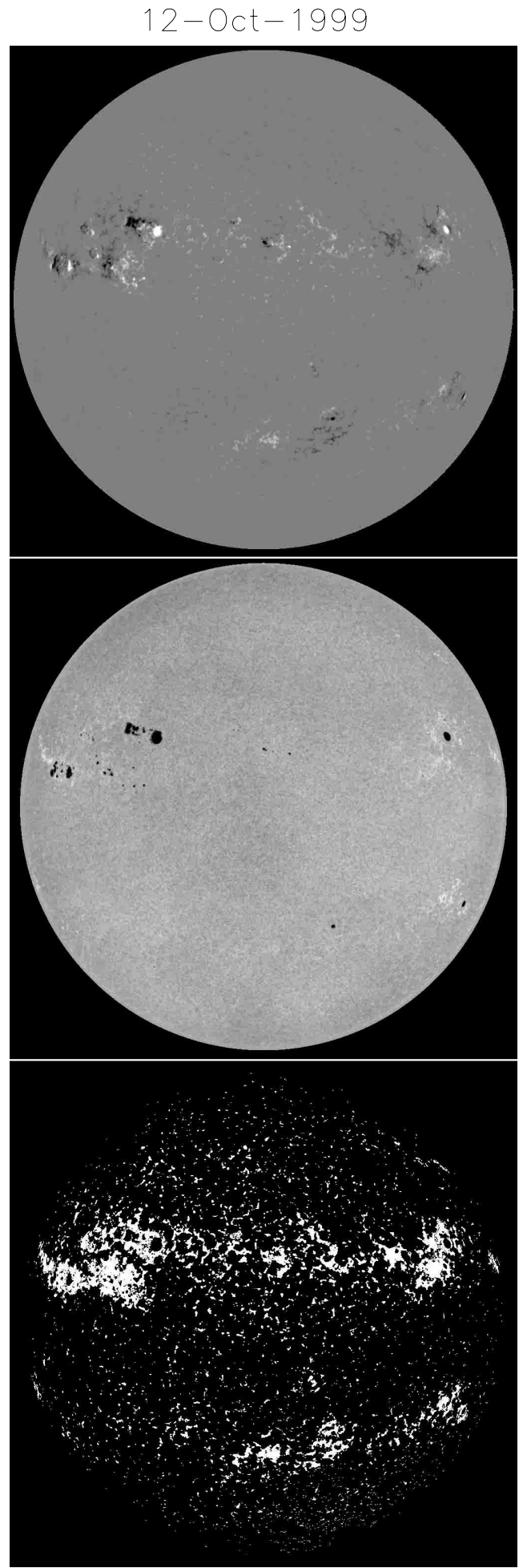

Fig. 1. Example of a $20 \mathrm{~min}$ averaged MDI magnetogram (top panel), the corresponding intensity image after removal of limb-darkening (middle panel) and the resulting contrast mask (lower panel) for October 12, 1999.

After that, their median was determined to eliminate the possible remaining activity. A second order surface was then fitted to the result and extrapolated to cover the whole solar disk. The resulting noise level, $\sigma_{\text {mag }}$, shows an increase towards the SW limb that probably includes

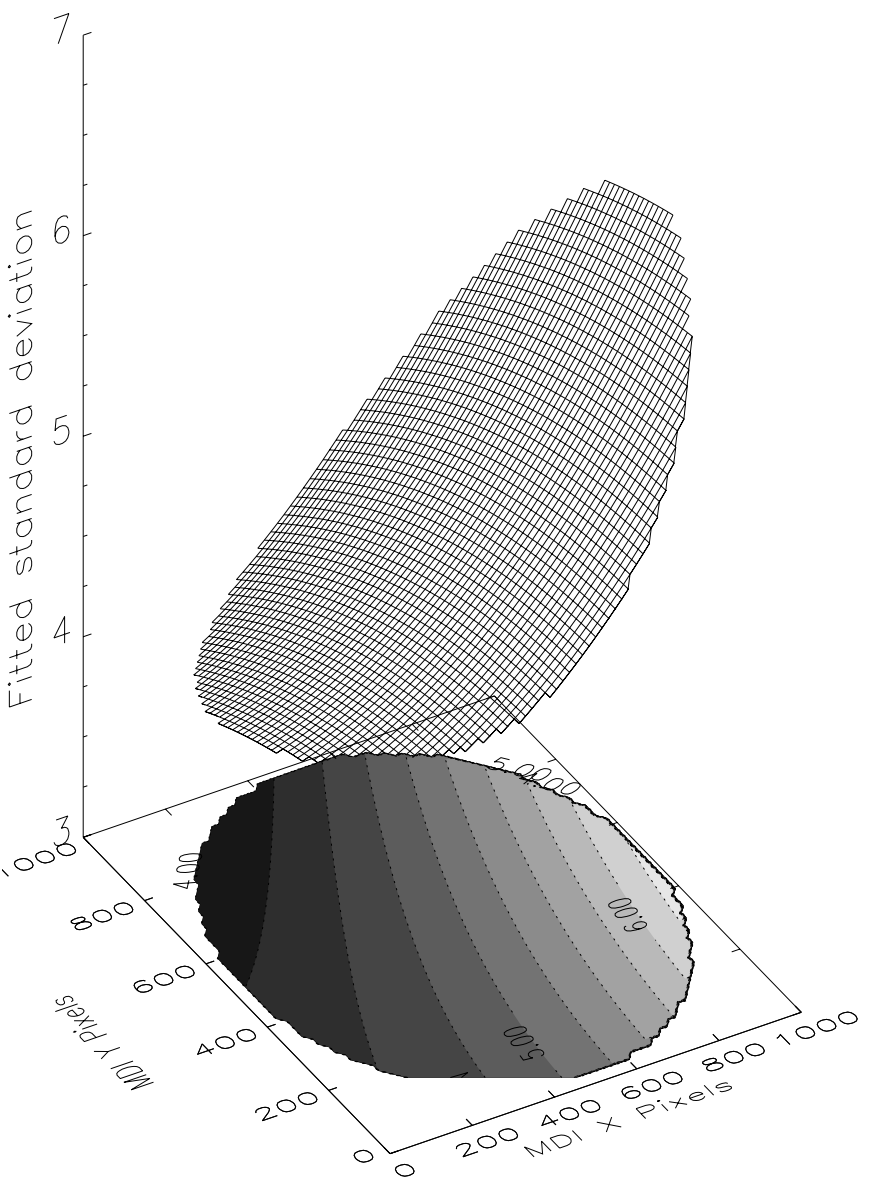

Fig. 2. Standard deviation (in G) of the 20-min averaged MDI magnetograms. An increase of the noise in the direction of the SW limb is evident. The shadowed contours indicate some of the values. Both the surface and the contours represent the standard deviation.

some velocity signal leakage. In Fig. 2 we show the calculated standard deviation for the 20-min averaged magnetograms. Note that when applying this noise surface to our data we have assumed that the MDI noise level has remained unchanged between 1996 and 1999.

A similar procedure has been used to determine the mean and standard deviation of the quiet Sun continuum intensity for each selected day, $\left\langle I_{\mathrm{qs}}\right\rangle$ and $\sigma_{I \mathrm{qs}}$ respectively, where the subscript qs denotes "quiet Sun". Every pixel in the running mean box with an absolute magnetic signal value below 0.5 times $\sigma_{\text {mag }}$ (i.e. pixels with corresponding magnetogram signal between approximately -2.5 and $2.5 \mathrm{G}$ ) has been considered as a quiet Sun pixel.

The surface distribution of solar magnetic features that produce a bright contribution to irradiance variations, is identified by setting two thresholds to every magnetogram-intensity image pair. The first threshold looks for magnetic activity of any kind, and is set to $\pm 3 \sigma_{\text {mag }}$, which corresponds, on average, to $15 \mathrm{G}$. As we are only interested in bright magnetic features, the second threshold masks out sunspots and pores by setting all pixels with a continuum intensity $3 \sigma_{\text {Iqs }}$ below the average 
to a null value. To reduce false detections, even at the risk of missing active pixels, we reject all isolated pixels above the given thresholds assuming that they are noise. $3 \times 10^{4}$ out of $10^{7}$ analyzed data points are rejected in this way. After this step, we find that $6 \%$ of the pixels satisfy both criteria. Using both thresholds we construct a mask of the contrast of bright features for each day. The result of applying the mask derived from the magnetogram (top panel) and intensity image (middle panel) shown in Fig. 1, is displayed in the bottom panel of that figure. Note that only features that lie above the given thresholds in the magnetogram and the intensity image are indicated by white pixels. Sunspots near the NE limb, for example, do not appear in the mask, but faculae surrounding those sunspots are well identified. Smaller features belonging to the magnetic network are also pinpointed outside of the active regions, although weaker elements of the network may well be missed. For each pixel with coordinates $(x, y)$, the contrast $C_{\text {fac }}$ is defined as:

$C_{\mathrm{fac}}(x, y)=\frac{I(x, y)-\left\langle I_{\mathrm{qs}}\right\rangle(x, y)}{\left\langle I_{\mathrm{qs}}\right\rangle(x, y)}$.

Contrast, magnetic field strength averaged over the pixel and position, represented by the heliocentric angle $\mu=$ $\cos (\theta)$, are calculated for each selected pixel. Finally, for each of these parameters the pixels above the thresholds for each of the 10 selected days are put together into vectors of about $6 \times 10^{5}$ elements, which should provide adequate statistics for a detailed study of the facular and network contrast.

The method used in this work resembles that employed by Topka et al. (1992, 1997), although our magnetic threshold is much lower due to the less noisy magnetograms used. The angular resolution, however, is also considerably lower, but it is constant.

\section{Results}

We have analyzed the AR faculae and network contrast dependence on both $\mu$ and the measured magnetic signal, $B$. It is important to recall that the observed magnetic signal drops to zero at the limb, even if strong magnetic field regions are present. This is a straightforward consequence of the fact that magnetograms are only sensitive to the line-of-sight component of the magnetic field and that the magnetic field is mainly vertical. To compensate this effect to first order we have worked with $B / \mu$ (i.e. $\langle|\boldsymbol{B}| \cos \gamma\rangle / \cos \theta)$ instead of $B$. Second order effects due to radiative-transfer effects or finite thickness of flux tubes remain.

We have binned the $B / \mu$ values into eight intervals that range from the threshold level of, on average, $15 \mathrm{G}$ to about $600 \mathrm{G}$. The intervals have been chosen so that they roughly contain the same number of pixels each, with the exception of the highest $B / \mu$ bins which have fewer pixels. By sorting the magnetic field strength into different bins we can distinguish between the CLV of the magnetic features present in regions with different filling factor $\alpha$.
Figure 3 displays the contrast, $C_{\text {fac }}$, as a function of $\mu$, individually for every $B / \mu$ interval. A second order polynomial has been fitted (employing least squares) to guide the eye and a dashed line indicating $C_{\text {fac }}=0$ has been included for clarity. To avoid overcrowding we have binned data points in sets of 40 before plotting.

Figure 3 reveals a clear evolution of the behaviour of the contrast from one $B / \mu$ interval to another. Network features (top left panel) show a low and almost constant contrast, as compared with the very pronounced CLV of the contrast for active region faculae (bottom panels). Intermediate cases show a progressive increase of the contrast towards the limb as well as an increasingly pronounced CLV. When $B / \mu<200 \mathrm{G}$, the contrast peaks at $\mu \sim 0.5$, while for magnetic signals $B / \mu \geq 200 \mathrm{G}$ this maximum shifts to lower values of $\mu$ (see Fig. 8 and its discussion in the text for a more quantitative analysis). Note also that for $B / \mu \geq 200 \mathrm{G}$ the contrast is negative around disk center, while it is positive in the network (i.e. for smaller $B / \mu$ ). We will return to this point in Sect. 4 . The large fluctuations of the contrast near the limb for intermediate and high magnetic signals are due to the distribution of active regions on the ten selected days.

Figure 4 shows the contrast as a function of $B / \mu$, for different positions on the solar disk. The solar disk has been divided into eight bins of $\mu$, centred on $\mu$ values ranging from 0.96 (disk center, top left) to 0.3 (limb, bottom right). Note that to keep the number of points in each bin approximately equal, $\mu$-bins lying closer to the limb are wider than the ones around $\mu=1$, showing therefore some overlap. Each point of the figure is obtained by binning together 40 points of data with similar $B / \mu$, and second degree polynomials have also been plotted, as in Fig. 3.

This figure shows that, in general, the contrast initially increases with $B / \mu$ before decreasing again. We expect it to continue decreasing for even larger values of $B / \mu$ representing pores and sunspots. At large $\mu$ the initial increase is small and the contrast basically decreases with $B / \mu$, while at small $\mu$ it mainly increases. For $\mu=1$, points with large $B / \mu$ show a negative contrast (as in the lower panels of Fig. 3), while points at the limb always have positive contrasts. The bins with $\mu>0.82$ do not display data for high magnetic signals, because their intensity is below the intensity threshold.

Given the regular behaviour of the contrast as a function of $\mu$ and magnetogram signal, $C_{\text {fac }}(\mu, B / \mu)$, it seems appropiate to search for an analytical expression for this dependence. We have performed a multivariate analysis using a $(\mu, B / \mu)$ grid. The $\mu$ values have been binned linearly, with $\Delta \mu=0.1 . B / \mu$ bins have been chosen to be equally spaced on a logarithmic scale, with $\Delta \log (B / \mu)=$ 0.05 , in order to compensate for the fact that magnetic signals are mostly concentrated towards lower values (Figs. 3 and 4$)$. The dimensions of the grid are $0.1 \leq \mu \leq 1$ and $17 \mathrm{G} \leq(B / \mu) \leq 630 \mathrm{G}$, resulting in a $9 \times 31$ bins grid. We do not consider points with $B / \mu>600 \mathrm{G}$ to exclude bright features that might belong to pores observed near 


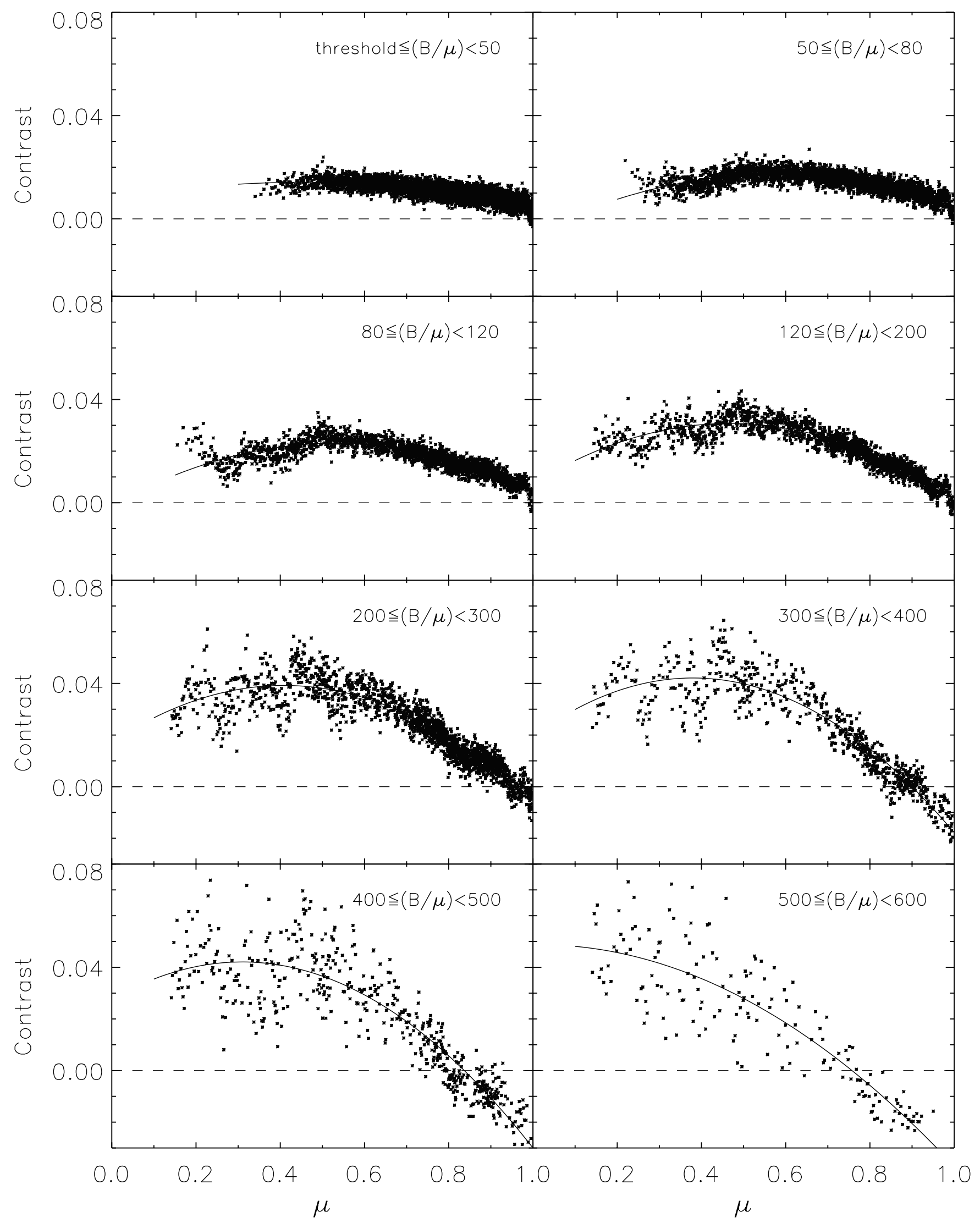

Fig. 3. Facular and network contrast as a function of $\mu$ for eight intervals of the strength of the magnetic field, from network values (top left panel) to strong faculae (lower right). A dashed line at $C_{\mathrm{fac}}=0$ has been plotted. The solid curves represent a second order polynomial least-squares fit to the points. Every dot represents 40 data points. $\mu=1$ is the disk center; $\mu=0$ is the limb. 


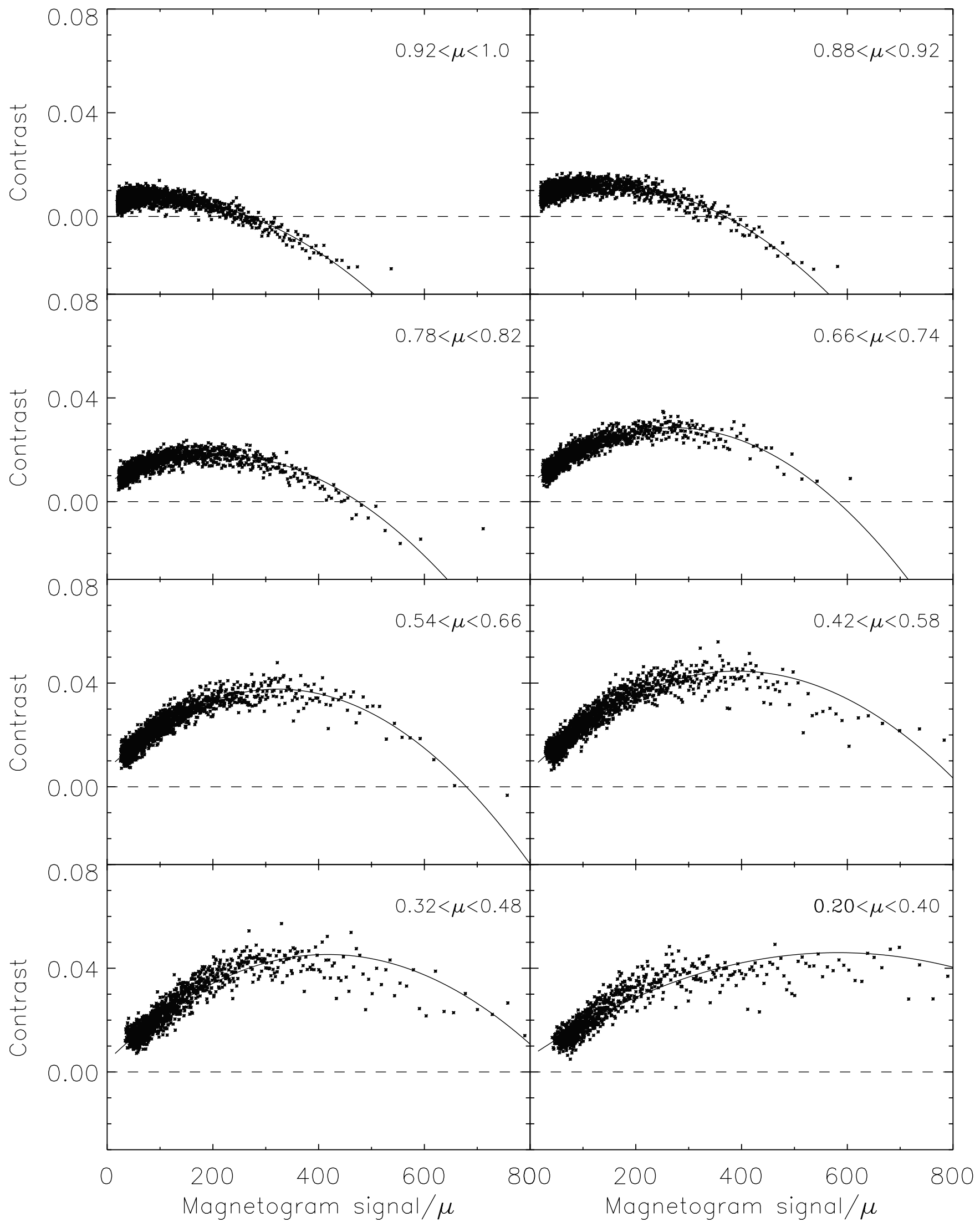

Fig. 4. Dependence of the contrast on the absolute value of the magnetogram signal, corrected for foreshortening effects. The solar disk has been divided into eight bins, from center to limb. Note that some $\mu$-bins overlap (see text for details). As in Fig. 3, a dashed line at $C_{\mathrm{fac}}=0$ has been plotted and solid curves represent a second order polynomial regression. Every dot represents 40 data points. 
the limb. We are aware of the fact that $600 \mathrm{G}$ is an arbitrary value for such a cutoff.

Each bin of the grid is defined by the averaged values of the contrast, $\mu$ and $B / \mu$, over all the data points of that bin. Although the curves in Fig. 3 are only intended to guide the eye, they do reveal that second order polynomials fit the contrast as a function of $\mu$ well. We have fitted the bidimensional array of contrasts by a second order polynomial function of $\mu$ and a cubic function of $B / \mu$ of the form:

$C_{\mathrm{fac}}(\mu, B / \mu)=\sum_{i, j} a_{j, i} \mu^{j}\left(\frac{B}{\mu}\right)^{i}$,

where $i$ runs from 1 to $3, j$ runs from 0 to 2 and $a_{j, i}$ are the coefficients of the fit. The result of the fit is a surface of second order in position $\mu$ and third order in magnetic signal. The coefficients of the multivariate fit $a_{j, i}$ are:

$$
\begin{aligned}
C_{\mathrm{fac}}(\mu, B / \mu)= & 10^{-4}\left[0.48+9.12 \mu-8.50 \mu^{2}\right]\left(\frac{B}{\mu}\right) \\
& +10^{-6}\left[0.06-2.00 \mu+1.23 \mu^{2}\right]\left(\frac{B}{\mu}\right)^{2} \\
& +10^{-10}\left[0.63+3.90 \mu+2.82 \mu^{2}\right]\left(\frac{B}{\mu}\right)^{3} .
\end{aligned}
$$

The terms of Eq. (2) are grouped in Eq. (3) to make clearer the quadratic dependence of $C_{\mathrm{fac}}(\mu, B / \mu)$ on $\mu$ and the cubic dependence on $B / \mu$. When $B / \mu$ is small $(<100 \mathrm{G})$, the first order term in $B / \mu$ provides the dominant contribution. When $B / \mu$ is large $(\geq 200 \mathrm{G})$, first and second order terms in $B / \mu$ dominate the contrast, modulated by the contribution of the cubic term which plays a role in this range of magnetic signals. At disk center $(\mu=1)$, those terms result in a dominant negative contribution (Fig. 3). Note that the contrast is constrained to go through zero when $B / \mu=0$, as expected for the quiet Sun.

The best-fit surface is shown in Fig. 5. The grid corresponds to the linear $\mu$-bins and the logarithmic $B / \mu$-bins taken for the fit. The shape of this surface is quite congruent with that shown by the observed contrast in Figs. 3 and 4. Note that the function given in Eq. (3) is valid only for the wavelength and spatial resolution of the MDI data, $6768 \AA$ and $2^{\prime \prime}$, respectively. For other values of these parameters we expect another dependence on $\mu$ and $B / \mu$. In particular, the absolute value of the contrast is expected to change.

To better estimate how this analytical surface fits the behaviour of the measured contrasts, we have sliced the surface in both directions, $\mu$ and magnetograph signal, and then compared the result with the measured values. In Fig. 6 the fitted surface is sliced along the $\mu$-axis (solid curve), at three sample magnetic signal ranges, representative of low (top panel), medium (middle panel) and high (lower panel) $B / \mu$ values. Dots represent measured contrasts. To avoid very crowded plots each dot represents 250 (top panel), 100 (middle) and 25 (bottom) data points, respectively. The different amounts of binning reflect the

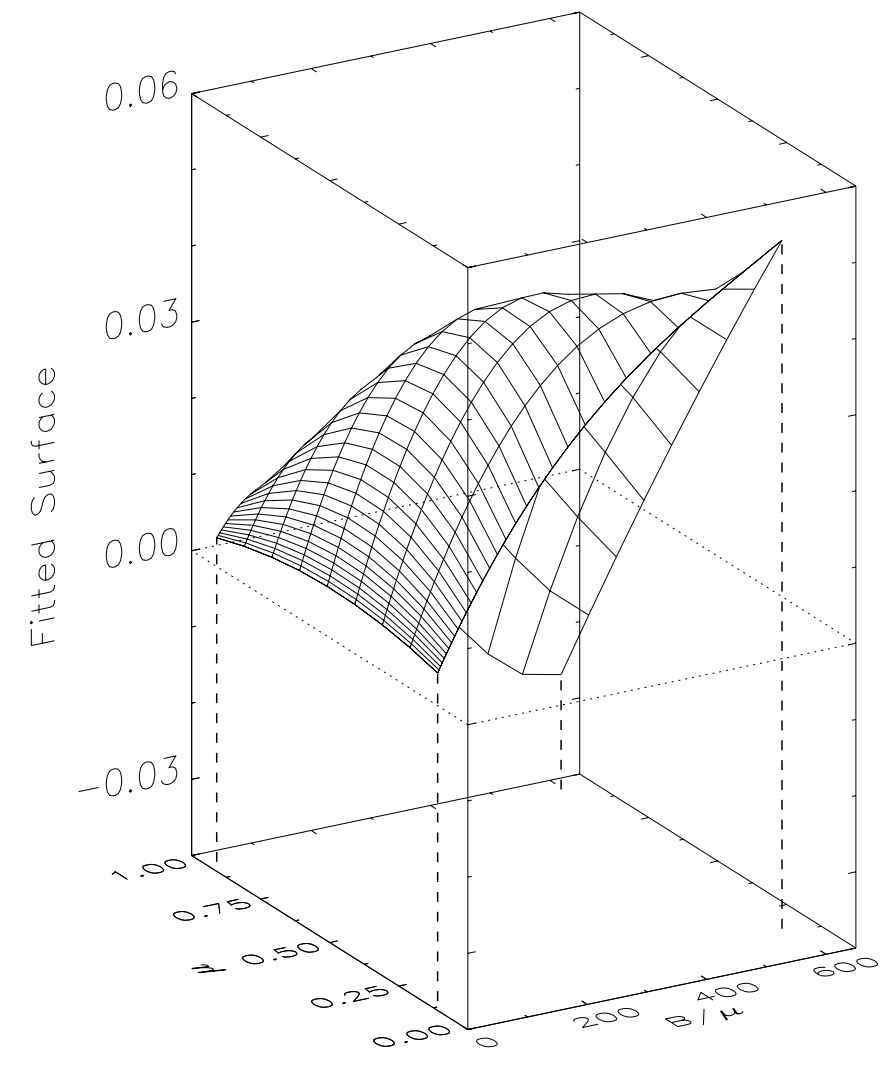

Fig. 5. Polynomial surface of second order in $\mu$ and third order in $B / \mu$ obtained from a multivariate fit performed to the grid of contrasts, covering $\mu$ and $B / \mu$ values. Dashed vertical lines project the corners of the plotted surface onto the $\mu-B / \mu$ plane and indicate the region spanned by the fit.

non-uniform distribution of points over the $B / \mu$ range. The multivariate regression surface fits quite well the plotted dependence of the contrast, although minor deviations are visible at small $B / \mu$. Figure 7 shows slices of the modeled surface along the $B / \mu$ axis (solid curves) and the corresponding binned data (dots), at three sample positions on the solar disk, from disk center (top panel) to near the limb (lower panel). The fitted curves now deviate somewhat more from the data points, most significantly for $0.54<\mu<0.66$, where the discrepancy can reach 0.01 in contrast.

The multivariate analysis yields to an expression for the contrast of photospheric bright features, $C_{\mathrm{fac}}(\mu, B / \mu)$, that cannot be directly compared with previous studies because, to our knowledge, no similar work has been done before. Quadratic functions have already been used by other authors (e.g. Foukal 1981) to fit the dependence of the facular contrast on position over the disk, although most of them use a function of the form $C_{\text {fac }}(\mu)=$ $b(1 / \mu-a)$ (Chapman 1980). A quadratic function agrees quite well with the CLV proposed by the hot wall model. A cubic function has been used for fitting the dependence of the contrast on magnetic strength. In this case we do not have a physical reason, only the goodness of the fit with respect to other bivariate functional dependences tried 


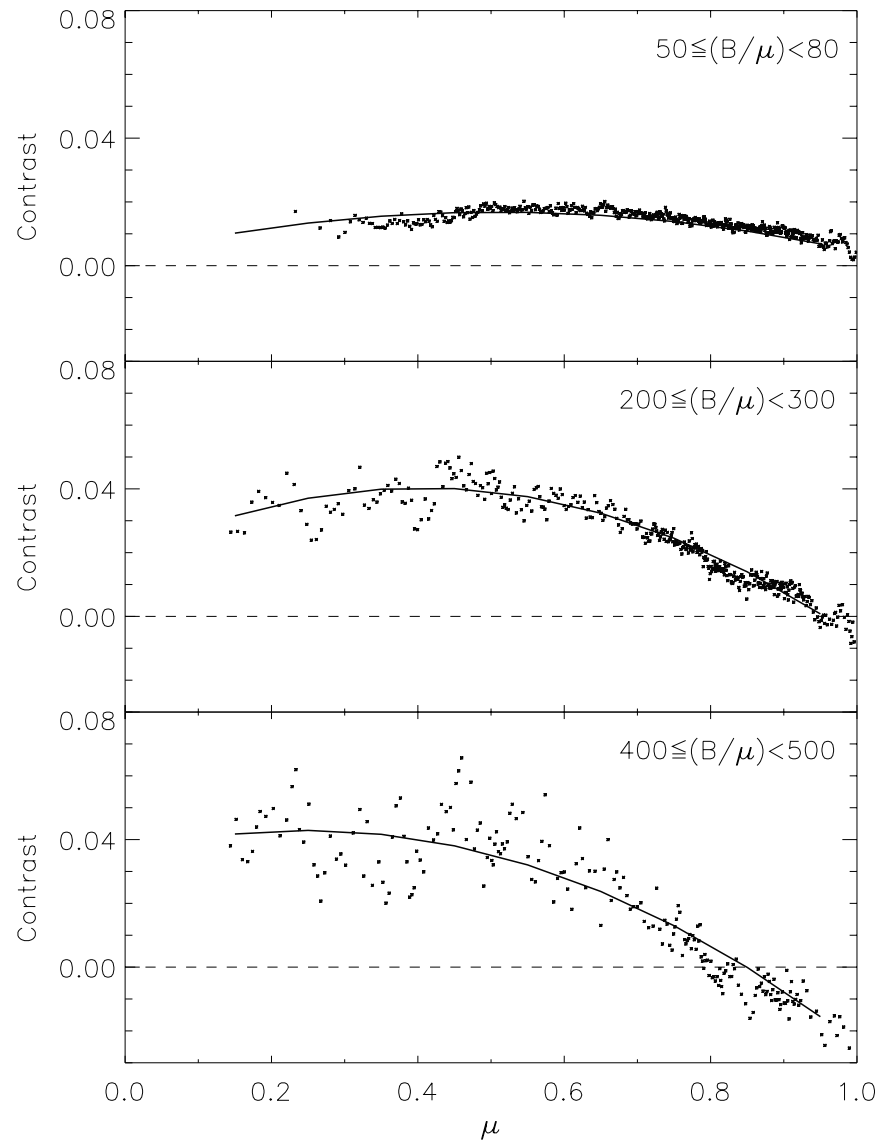

Fig. 6. Comparison of cuts through the surface (solid curves) returned by the multivariate analysis and the measured contrasts (dots) as a function of $\mu$, for 3 sample bins of corrected magnetic signal. Every dot represents 250 (top), 100 (middle) and 25 (bottom) data points.

(see Figs. 6 and 7) and the requirement to force the contrast through zero for a disappearing magnetic signal. We suspect that, in order to obtain a better empirical description of the dependence of facular contrast on $\mu$ and $B / \mu$, a larger number of free parameters is required.

The dependence of the peak of $C_{\mathrm{fac}}$ on $B / \mu$ is shown in Fig. 8 . The $\mu$-values at which $C_{\mathrm{fac}}$ peaks, $\mu_{\max }$, have been represented against the corresponding magnetic signal in Fig. 8a. Figure $8 \mathrm{~b}$ shows the peak $C_{\mathrm{fac}}$ values reached, $C_{\mathrm{fac}}^{\max }$ (see Fig. 3), plotted as a function of $B / \mu$. Finally Fig. 8c shows $C_{\mathrm{fac}}^{\max } /(B / \mu)$ plotted against $B / \mu$ or, in other words, the dependence on the magnetic signal of the specific contrast per unit of magnetic flux. Errors in $\mu$ are estimated from the difference between the peak of the best-fit curves in Fig. 3 and the peak obtained directly from the data points. Error bars in $B / \mu$ correspond to the size of the $B / \mu$-intervals used in Fig. 3 .

A linear regression adequately describes the dependence of $\mu_{\max }$ on $B / \mu$ for the precision achievable with the current data. The best fit straight line (solid line in Fig. 8a) is

$\mu_{\max }=5.60 \times 10^{-1}-4.97 \times 10^{-4}(B / \mu)$.

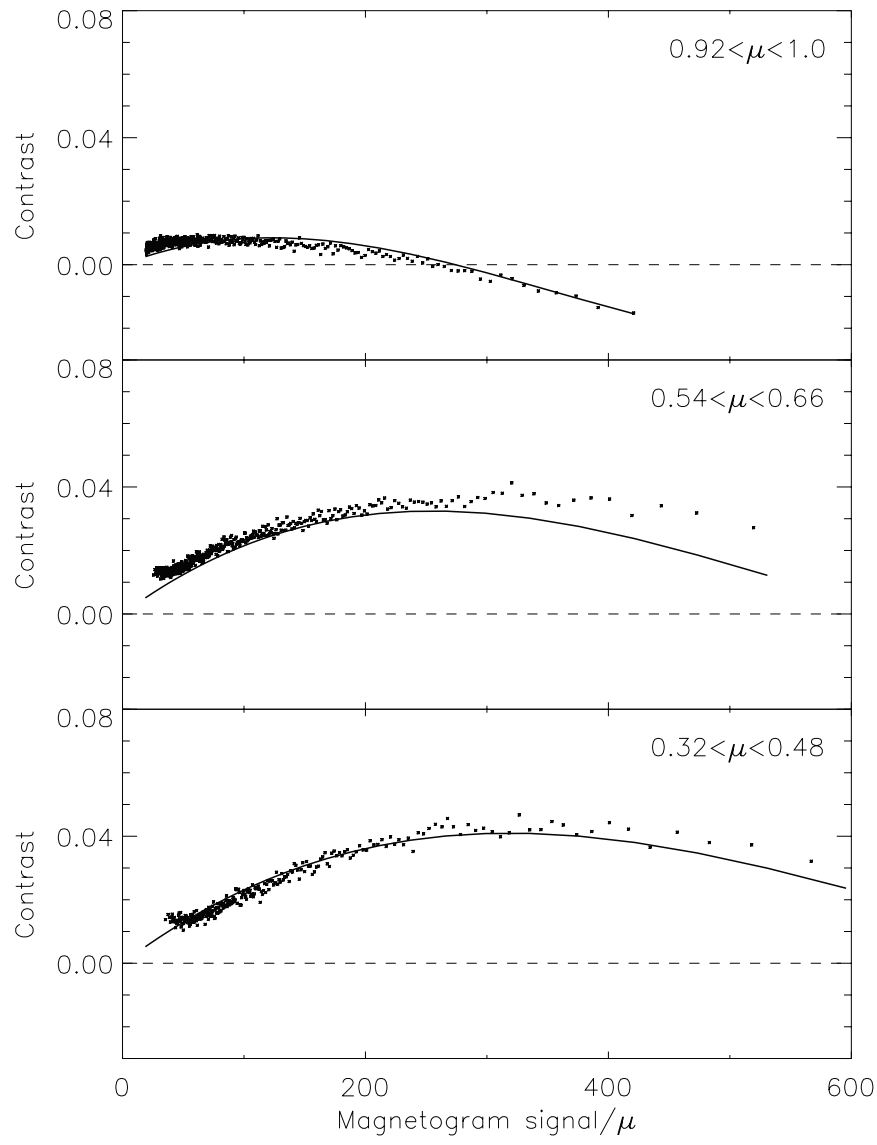

Fig. 7. The same as Fig. 6 , but for cuts along the $B / \mu$-axis (solid curves) made at three positions on the solar disk. Dots represent measured contrasts. The plotted curves represent the same $\mu$ ranges as those of the data points. Every dot represents 200 data points.

Thus, $\mu_{\max }=0.56 \pm 0.02$ when $B / \mu$ tends to zero, and $\mu_{\max }=0$ for $B / \mu \approx 1120 \mathrm{G}$ from extrapolations of this curve. Figure $8 \mathrm{c}$ implies that the contrast per unit of magnetic signal decreases strongly with increasing magnetogram signal. Since individual flux tubes are not resolved by MDI, we cannot infer the intrinsic contrast of a flux tube from Fig. 8b, which obviously shows the same pattern as Fig. 4. However, by normalizing by $B / \mu$ we obtain a quantity that is roughly proportional to the intrinsic brightness of the flux tubes (assuming that the field strength of the elemental magnetic flux tubes lies in a narrow range as mentioned in Sect. 2).

\section{Discussion}

\subsection{Comparison with previous observations}

Comparison with other contrast observations is not easy because of the differences in the selected wavelength, spatial resolution, range of studied heliocentric angles, magnetic filling factor and size of the analyzed features. All these factors contribute to the scatter between the existing contrast measurements. 

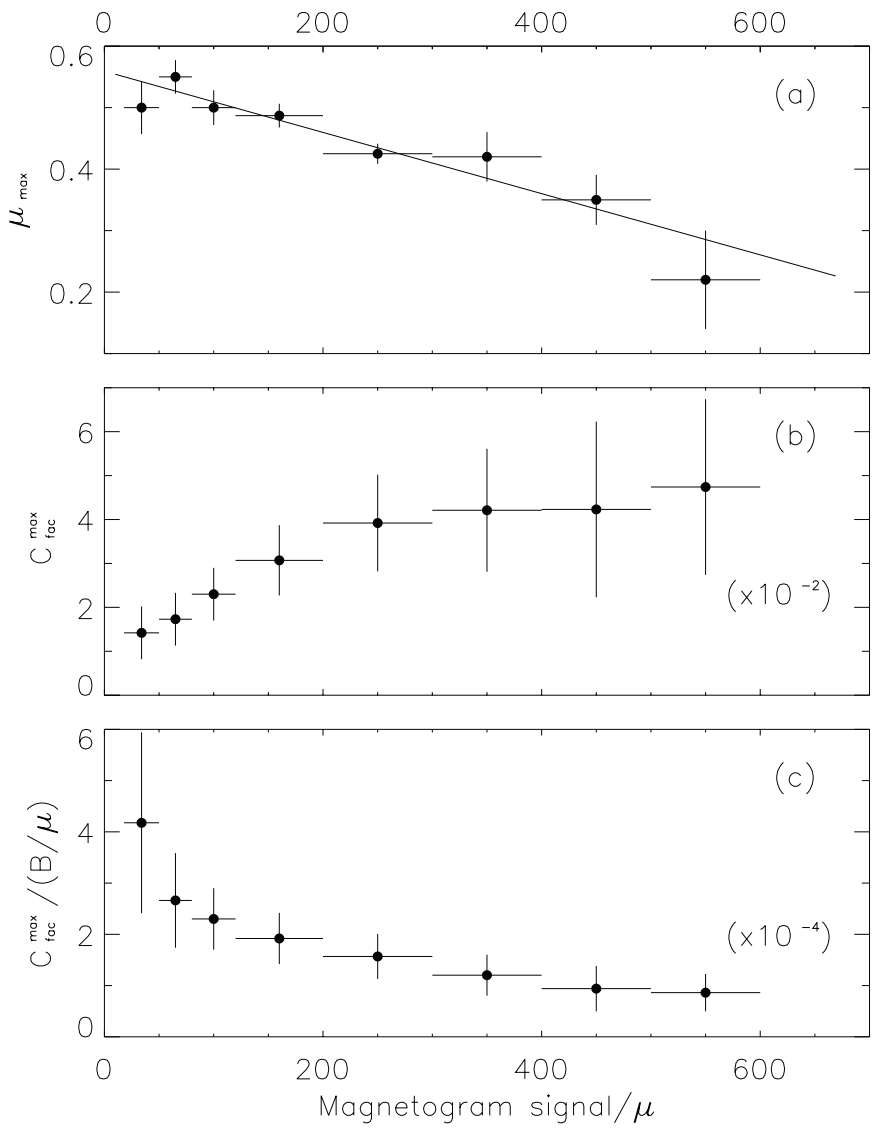

Fig. 8. Dependence on the magnetic flux per pixel, $B / \mu$, of: a) $\mu_{\max }$; b) $C_{\mathrm{fac}}^{\max }$ times $\left.10^{2} ; \mathbf{c}\right) C_{\mathrm{fac}}^{\max } /(B / \mu)$ times $10^{4}$. See text for details.

Our results differ from earlier observations of the contrast of bright features, specially when considering magnetic signals $B / \mu>200 \mathrm{G}$ at disk center. Previous measurements of disk center facular contrast have frequently yielded positive values, although they usually were close to zero. Thus, from multi-color photometric images, Lawrence (1988) measures $C_{\text {fac }} \sim 0.005$ at disk center, and Lawrence et al. (1988) find $C_{\mathrm{fac}}=0.007 \pm 0.001$. In fact, our results agree better with those of Topka et al. (1992, 1997) and Lawrence et al. (1993) despite the difference in spatial resolution and studied wavelength. For $200 \mathrm{G} \leq(B / \mu) \leq 600 \mathrm{G}$ the agreement is also surprisingly good. Nevertheless, these authors distinguish between active regions and quiet Sun. For active regions they always measure a negative contrast around disk center (for $\mu=0.97$ and $\mu=0.99$ ) irrespective of the magnetogram signal, while we get slighly positive contrast values for $B / \mu \leq 200 \mathrm{G}$ in agreement with their results for the network. Since at these field strengths most of our signal originates in the network, this agreement is probably not surprising.

Chapman \& Klabunde (1982) claim that the contrast shows a sharp increase near the limb (and even fit a $\mu^{-1}$ dependence). We find that $C_{\text {fac }}$ peaks between $\mu=0.5$ and $\mu=0.2$, depending on the magnetic strength of the signal, and then decreases towards the limb (Fig. 3). Libbrecht
\& Kuhn $(1984,1985)$ also find this behaviour; however, they give $\mu \leq 0.2$ for the peak of the contrast. Wang \& Zirin (1987) and Spruit's hot wall model also give a similar value for the $\mu$ at which $C_{\text {fac }}$ peaks. It is worth noting that Libbrecht \& Kuhn $(1984,1985)$ and Wang \& Zirin (1987) do not take into account the magnetic field of the observed feature, which makes the comparison between our results and theirs more difficult, as the CLV obtained is different when features are selected according to their brightness rather than the magnetogram signal. In the former case there is a bias towards brighter features. Our results indicate that the higher the magnetic signal, the smaller the $\mu$-value at which the contrast peaks (see Fig. 8a) so that network-like features dominate at disk center and features with increasingly large $B / \mu$ closer to the limb. This should move the peak of the contrast to smaller $\mu$ when the brightest features are searched for, than when magnetograms are used to identify faculae. Finally, it should be pointed out that, for increasingly smaller $B / \mu$ values the contrast becomes increasingly independent of $\mu$; this agrees with the conclusion of Ermolli et al. (1999) that the network contrast is almost independent of $\mu$.

It is remarkable that an expression as given by Eq. (2) reproduces the dependence of the contrast of bright features on their position $(\mu)$ and on the magnetic flux per pixel $(B / \mu)$, within the range $0.1 \leq \mu \leq 1$ and $17 \mathrm{G} \leq(B / \mu) \leq 630 \mathrm{G}$. A relative accuracy of better than $10 \%$ is achieved almost everywhere within this domain. However, this multivariate analysis is only a first step and considerable further work needs to be done, since two other relevant parameters for the contrast, namely the wavelength and the spatial resolution, are kept at fixed values (those prescribed by MDI) in our analysis. A 4-dimensional data set is thus needed. A first step was taken by Lawrence et al. (1993), who compared observations from different instruments. At least some further progress in this direction can be achieved by employing MDI high resolution data, although off-center pointing is required.

\subsection{Comparison with flux-tube models}

MHD models including self-consistent energy transfer predict that small flux tubes (diameters smaller than $300 \mathrm{~km}$ ) appear bright at disk center but with decreasing contrast near the limb; somewhat larger tubes are predicted to appear dark at disk center but bright near the limb, and finally, very large flux tubes (pores and sunspots; not considered in this study) are predicted to be dark everywhere (e.g., Knölker \& Schüssler 1988, 1989). In such models the contrast at $\mu \approx 0.1$ is largely determined by the brightness of the bottom of the flux tube (and the brightness of its surroundings, e.g. granular down flow lanes), while the CLV of the contrast is strongly influenced by the visibility of the hot walls. The bottom of a flux tube is defined as the horizontal optical depth unity surface in the interior of a flux tube. 
Our results are qualitatively in accordance with this prediction if we make two reasonable assumptions. First, the network and facular features are composed of a mixture of spatially unresolved flux tubes of different sizes. Second, the average size of the flux tubes increases with increasing magnetogram signal or filling factor. Under these assumptions the upper panels of Fig. 3 refer to, on average, small flux tubes which dominate the network, while the lower panels of that figure refer to larger tubes mostly present in AR faculae. In our study the contrast always has a minimum at $\mu=1$ and increases with decreasing $\mu$ (as part of the hot wall becomes visible), until a maximum when the contrast peaks (the maximum surface of the hot wall is seen). Closer to the limb the contrast decreases as less wall surface is exposed. There are, however, clear differences between small $B / \mu$ network flux tubes and tubes found in $\mathrm{AR}$ faculae, i.e. regions with large $B / \mu$. Network tubes are bright everywhere on the solar disk and exhibit a low contrast (Fig. 8b), but a high specific contrast (Fig. 8c). This implies that network flux tubes are brighter than AR flux tubes and partly reflects the fact that network flux tubes are hotter than AR tubes (e.g., Solanki \& Brigljević 1992; Solanki 1993). The greater brightness at large $\mu$ implies that network flux tubes have a hotter bottom than larger flux tubes. Since this is also true at $\mu \leq 0.6$, it suggests that the walls of smaller tubes, or of tubes in regions with lower filling factor, are hotter as well. This is in agreement with the theoretical finding of Deinzer et al. (1984b) that the inflow of radiation into the tube leads to a cooling of the surroundings and a lowering of the temperature of the walls. This temperature reduction is indeed predicted to be greater for larger flux tubes (Knölker \& Schüssler 1988).

A mixture of flux-tube sizes at a given $B / \mu$ is needed because the CLV of $C_{\text {fac }}$ at small $B / \mu$ does not agree with the predictions for any size of flux tube. The model flux tubes are all bright over only a relatively small range of $\mu$ values. Hence the mixture of flux tube sizes is needed in order to produce a relatively $\mu$-independent contrast, as exhibited by magnetic features at small $B / \mu$. As can be seen in Fig. 3, the contrast shows a more pronounced CLV as tube size increases, in accordance with the hot wall model, and larger tubes have a negative contrast at disk center, as predicted. The high specific contrast of small $B / \mu$ features (Fig. $8 \mathrm{c}$ ), and the fact that their contrast is positive over the whole solar disk indicates that a change in the magnetic flux of the network has a much larger contribution to the change of the irradiance than a similar change in flux in active regions.

From Fig. 8a we can determine the heliocentric angles that make the contrast peak, $\theta_{\max }$. For the intervals displayed on Fig. $3, \theta_{\max }$ is $63^{\circ}, 55^{\circ}, 58^{\circ}, 62^{\circ}, 66^{\circ}, 68^{\circ}, 72^{\circ}$ and $77^{\circ}$, respectively. Assuming the hot wall model with a simplified cylindrical geometry for the flux tubes, a Wilson depression $Z_{\mathrm{W}}$ of $150 \mathrm{~km}$ (Spruit 1976) and the derived $\theta_{\max }$ values, it is possible to roughly estimate the average value of the tube diameter for each magnetic range. Taking into account that the maximum depth of the wall
$Z_{\mathrm{W}}$ is seen when the angle between the local vertical to the tube and the line of sight is equal to the heliocentric angle, then the diameter $D$ should be $D=Z_{\mathrm{W}} \tan \left(\theta_{\max }\right)$. Applying this approximation to our observations, we obtain diameters of 290,210,240,281,334, 365, 460, and $650 \mathrm{~km}$ respectively, for the mentioned $\theta_{\max }$ values and their respective magnetic ranges. These diameters are estimated to be uncertain by approximately a factor of two. For example, uncertainties in $Z_{\mathrm{W}}$ translate into proportionate relative uncertainties in $D$.

Finally, we wish to draw attention to the wiggle of the measured contrasts around $\mu=0.95$ in Figs. 3 and 6 . This can be observed at all magnetic strengths. At $\mu=1$ the contrast has a minimum value, then increases, descending a bit later for still smaller $\mu$ 's before finally increasing slowly towards the limb. Topka et al. (1992) show some of these variations very close to disk center in Fig. 5 of their paper. They argue that such variations are partly due to the inclination of the flux tubes of opposite polarities toward each other in the active region they observe. However, we average over many network elements on multiple days and the persistence of such a structure is surprising, in particular also for small $B / \mu$ values where the statistics are extremely good.

\section{Conclusions}

The results presented in this work indicate that the CLV of the continuum contrast of magnetic features changes gradually with magnetogram signal (or magnetic filling factor), such that the contrasts of AR faculae and the network exhibit a very different CLV, in general agreement with the results of Topka et al. $(1992,1997)$ and Lawrence et al. (1993). A possible reason for the difference is that the populations of magnetic flux tubes found in the two kinds of features are, on average, different in size, in agreement with the conclusions of earlier investigations (GrossmannDoerth et al. 1994; Keller 1992).

Stronger magnetogram signals, corresponding to wider flux tubes on average (Grossmann-Doerth et al. 1994), appear dark at disk centre, but bright near the limb, while the weakest signals (on average narrower flux tubes) are almost equally bright at disk centre and near the limb. This result is in good agreement with the predictions of theoretical flux tube models (Deinzer et al. 1984a, 1984b; Knölker et al. 1988; Knölker \& Schüssler 1988) if there is a distribution of flux tube sizes present within an MDI pixel. Because network elements are bright over the whole solar disk their contribution to irradiance variations is significant and needs to be taken into account when reconstructing variations of the total solar irradiance.

One advantage of the present investigation relative to that of Topka et al. $(1992,1997)$ is that by using full disk MDI data we have a result for a very well defined spatial resolution, so that any models derived on the basis of these results can be directly used for reconstructing total and spectral solar irradiance, as for instance measured by VIRGO (Fröhlich et al. 1995), without further adjustment. 
A new result of this work is that, with a simple expression, we can predict the contrast of a bright feature, from network and small tubes to faculae of different sizes, given its position and magnetic strength within a certain range, and reproduce simultaneously the $C_{\mathrm{fac}}(\mu)$ and $C_{\mathrm{fac}}(B / \mu)$ dependences.

In a next step the dependence of the contrast on wavelength (for given $\mu$ and $B / \mu$ ) must be determined, as well as the dependence on spatial resolution. The later dependence is of particular interest also because the investigations of Lawrence et al. (1993) and Topka et al. (1997) give similar values of the contrast near $\mu=1$ as we find, although the spatial resolution of the La Palma data employed by these authors is almost an order of magnitude higher than that of the MDI full disk data $\left(0.5^{\prime \prime}\right.$ versus $\left.4^{\prime \prime}\right)$. Closer to the limb Topka et al. (1997) obtain contrasts a factor of two higher. Whether this is due to the different wavelengths observed or has another source needs to be investigated.

Acknowledgements. AO acknowledges financial support from the DURSI (Generalitat de Catalunya) grant 2001 TDOC 00021, as well as partial financial support from the Max-Planck-Institut für Aeronomie, from E. Ortiz and S. Carbonell, and from the European Space Agency (under contract ESA-ESTEC 14098/99/NL/MM). VD acknowledges partial financial support from the DURSI (Generalitat de Catalunya).

\section{References}

Balmforth, N. J., Gough, D. O., \& Merryfield, W. J. 1996, MNRAS, 278, 437

Berger, T. E., Schrijver, C. J., Shine, R. A., et al. 1995, ApJ, 454,531

Chapman, G. A. 1980, ApJ, 242, L45

Chapman, G. A. 1987, ARA\&A, 25, 633

Chapman, G. A., \& Klabunde, D. P. 1982, ApJ, 261, 387

Deinzer, W., Hensler, G., Schüssler, M., \& Weisshaar, E. 1984a, A\&A, 139, 426

Deinzer, W., Hensler, G., Schüssler, M., \& Weisshaar, E. 1984b, A\&A, 139, 435

Domingo, V., Fleck, B., \& Poland, A. I. 1995, Sol. Phys., 162, 1

Ermolli, I., Berrilli, F., \& Florio, A. 1999, in ESA SP-448, Proc. 9th European Meeting on Solar Physics, Magnetic Fields and Solar Processes (ESA Publications Division, Florence, Italy), 275

Fligge, M., Solanki, S. K., Meunier, N., \& Unruh, Y. 2000a, in ESA SP-463, The First SOLSPA Euroconference: The Solar Cycle and Terrestrial Climate (ESA Publications Division, Tenerife, Spain), 117

Fligge, M., \& Solanki, S. K. 2000b, JA\&A, 21, 275

Foukal, P. 1981, in Physics of Sunspots, ed. L.E. Cram, \& J.H. Thomas, Sacramento Peak Observatory, Sunspot, NM, 391

Foukal, P., \& Fowler, L. 1984, ApJ, 281, 442

Foukal, P., \& Lean, J. L. 1988, ApJ, 328, 347

Frazier, E. N. 1971, Sol. Phys., 21, 42
Fröhlich, C., Romero, J., Roth, H., et al. 1995, Sol. Phys., 162,101

Grossmann-Doerth, U., Knölker, M., Schüssler, M., \& Weisshaar, E. 1989, in NATO ASI Ser. C-263, Solar and Stellar Granulation, ed. R. J. Rutten, \& G. Severino (Kluwer, Dordrecht), 191

Grossmann-Doerth, U., Knölker, M., Schüssler, M., \& Solanki, S. K. 1994, A\&A, 285, 648

Keller, C. U. 1992, Nature, 359, 307

Knölker, M., \& Schüssler, M. 1988, A\&A, 202, 275

Knölker, M., \& Schüssler, M. 1989, in Flux tubes in the solar atmosphere, ed. E. Leer and P. Maltby, Institute of Theoretical Astrophysics (University of Oslo), 17

Knölker, M., Schüssler, M., \& Weisshaar, E. 1988, A\&A, 194, 257

Kuhn, J. R., Libbrecht, K. G., \& Dicke, R. H. 1988, Science, 242, 908

Lawrence, J. K. 1988, Sol. Phys., 116, 17

Lawrence, J. K, Chapman, G. A., \& Herzog, A. D. 1988, ApJ, 324,1184

Lawrence, J. K., Topka, K. P., \& Jones, H. P. 1993, J. Geophys. Res., 98, 18911

Lean, J. L., Cook, J., Marquette, W., \& Johannesson, A. 1998, ApJ, 492, 390

Libbrecht, K. G., \& Kuhn, J. R. 1984, ApJ, 277, 889

Libbrecht, K. G., \& Kuhn, J. R. 1985, ApJ, 299, 1047

Muller, R. 1983, Sol. Phys., 87, 243

Neckel, H., \& Labs, D. 1994, Sol. Phys., 153, 91

Scherrer, P. H., Bogart, R. S., Bush, R. I., et al. 1995, Sol. Phys., 162, 129

Solanki, S. K. 1986, A\&A, 168, 311

Solanki, S. K. 1993, Space Sci. Rev., 61, 1

Solanki, S. K. 1994, in The Sun as a Variable Star: Solar and Stellar Irradiance Variations, ed. J.M. Pap, C. Fröhlich, H. Hudson, \& S.K. Solanki (Cambridge Univ. Press), IAU Coll., 143, 226

Solanki, S. K. 2001, in Magnetic Fields Across the HR Diagram, ed. G. Mathys, S.K. Solanki, \& D. Wickramasinghe, Astronomical Society of the Pacific, ASP Conf. Ser., 248, 45

Solanki, S. K., \& Brigljević, V. 1992, A\&A, 262, L29

Solanki, S. K., Finsterle, W., Rüedi, I., \& Livingston, W. 1999, A\&A, 347, L27

Solanki, S. K., \& Fligge, M. 2001, Adv. Space Res., in press

Solanki, S. K., \& Stenflo, J. O. 1984, A\&A, 140, 185

Spruit, H. C. 1976, Sol. Phys., 50, 269

Spruit, H. C., \& Zwaan, C. 1981, Sol. Phys., 70, 207

Steiner, O., Grossmann-Doerth, U., Schüssler, M., \& Knölker, M. 1996, Sol. Phys., 164, 223

Topka, K. P., Tarbell, T. D., \& Title, A. M. 1992, ApJ, 396, 351

Topka, K. P., Tarbell, T. D., \& Title, A. M. 1997, ApJ, 484, 479

Unruh, Y. C., Solanki, S. K., \& Fligge, M. 1999, A\&A, 345, 635

Wang, H., \& Zirin, H. 1987, Sol. Phys., 110, 281

Willson, R. C., \& Hudson, H. S. 1988, Nature, 332, 810

Zayer, I., Stenflo, J. O., Keller, C. U., \& Solanki, S. K. 1990, A\&A, 239, 356 